UDC 51

\title{
Formal Diagonalisation of Lax-Darboux Schemes
}

\author{
A.V. Mikhailov \\ Received November 28, 2015
}

We discuss the concept of Lax-Darboux scheme and illustrate it on well known examples associated with the Nonlinear Schrödinger (NLS) equation. We explore the Darboux links of the NLS hierarchy with the hierarchy of Heisenberg model, principal chiral field model as well as with differential-difference integrable systems (including the Toda lattice and differential-difference Heisenberg chain) and integrable partial difference systems. We show that there exists a transformation which formally diagonalises all elements of the Lax-Darboux scheme simultaneously. It provides us with generating functions of local conservation laws for all integrable systems obtained. We discuss the relations between conservation laws for systems belonging to the Lax-Darboux scheme.

Keywords: formal diagonalisation, Lax-Darboux schemes, nonlinear Schrödinger equation, NLS

For citation: Mikhailov A. V., "Formal Diagonalisation of Lax-Darboux Schemes", Modeling and Analysis of Information Systems, 22:6 (2015), 795-817.

\section{On the authors:}

Alexander V Mikhailov - Professor, University of Leeds, School of Mathematics (Leeds, UK)

University of Leeds, Leeds, LS2 9JT, UK

e-mail: A.V.Mikhailov @ leeds.ac.uk 


\section{Introduction}

Often, integrable partial differential, differential-difference and partial difference equations can be regarded as parts of an algebraic structure which we call a Lax-Darboux scheme. In this context the Lax representations for the integrable partial differential equation (PDE) and a hierarchy of its symmetries form a Lax structure. Darboux transformations for the corresponding Lax operators are automorphisms of this Lax structure resulting in a chain of Bäcklund transformations for the PDE and its symmetries. The latter represents an integrable system of differential-difference equations (D $\Delta E s$ ) and its symmetries (often non-evolutionary). The Bianchi permutability conditions for Darboux transformations represent a system of partial difference equations (P $\Delta \mathrm{Es}$ ). This system possesses an infinite hierarchy of commuting symmetries (the above mentioned $\mathrm{D} \Delta \mathrm{Es}$ ) and thus it is an integrable partial difference system in its own right. There are many journal publications and monographs focusing on certain aspects of this big picture [1, 2, 3, 4, 5]. In particular, paper [4] contains a good collection of Lax-Darboux representations recursion operators for integrable differential-difference equations. In this paper we discuss the ways in which PDEs, D $\Delta$ Es and P $\Delta$ Es belonging to the same LaxDarboux scheme share the same hierarchy of local conservation laws.

The main idea standing behind our theory is a formal diagonalisation of the LaxDarboux scheme. We show that there exists a formal (i.e. in the form of a formal series) gauge transformation which simultaneously diagonalises (or brings to a block-diagonal form) the Lax operators of the Lax structure and Darboux matrices associated with Darboux transformations. It provides us with a regular method for recursive derivation of a hierarchy of local conservation laws for the nonlinear differential and difference systems associated with the Lax-Darboux scheme.

The method of formal diagonalisation of differential operators can be found in the classical literature concerning asymptotic expansion [6]. In application to Lax representations for partial differential equations and recursive derivation of the hierarchy of local conservation laws, there is a neat and instructive exposition of the method [7]. It has been successfully used in the Symmetry Approach to classify of integrable partial differential equations [8]. Here we extend the method to Darboux transformations and in this way to differential-difference and partial difference integrable systems. We shall explain the method using the Lax-Darboux scheme associated with the Nonlinear Schrödinger equation. Its generalisations to other Lax-Darboux schemes is rather straightforward. This paper is based on a lecture courses given by the author in the Bashkir State University (Ufa, 2012) and as a part of MAGIC course on Integrable systems (UK, 2014), a number of conference talks (Ufa, October 2012; Moscow, November 2012 [9]; Cambridge, July 2013 [10]) where the concept of Lax-Darbiux scheme and formal diagonalisation approach were originally presented. This method has proven to be useful in a many applications(see for example $[5,11,12]$ ). 


\section{Lax-Darboux scheme for the Nonlinear Schrödinger equation}

In this paper we consider Lax integrable equations, i.e. equations which can be integrated using the Inverse Spectral Transform method. For such equations we can build up a LaxDarboux scheme with the following objects:

- the Lax structure, which is an infinite sequence of Lax operators whose commutativity conditions are equivalent to the equation and a hierarchy of commuting symmetries;

- Darboux transformations, which are automorphisms of the Lax structure;

- Bäcklund transformations, which are follow from the compatibility conditions of the Lax structure and Darboux transformation. They can be regarded as integrable differential-difference systems;

- the conditions of Bianchi permutability for the Darboux transformations, which lead to systems of integrable partial difference equations;

- Ajacent Lax structures associated with a Darboux transformation which lead to adjacent symmetries of these differential-difference and partial difference equations and are integrable differential-difference equations in their own right.

In this section we would like to give explicit representations of all listed above objects in the case of the Nonlinear Schrödinger (NLS) equation.

\subsection{Lax structure of the Nonlinear Schrödinger Equation}

The Nonlinear Schrödinger Equation is a system of two partial differential equations

$$
2 p_{t}=p_{x x}-8 p^{2} q, \quad 2 q_{t}=-q_{x x}+8 q^{2} p
$$

where $x, t$ are independent variables. In the literature the term Nonlinear Schrödinger Equation usually stands for one complex equation of the form

$$
i q_{t}=q_{x x} \pm 2|q|^{2} q,
$$

which can be obtained from (1) after a change of variables $t \rightarrow 2 i t, x \rightarrow 2 i x$ and reduction $p=\mp q^{*}$. In this paper we shall use equation (1) to illustrate the method, since the reduction condition would add some inessential technicalities.

It has been shown by Zakharov and Shabat [13] that the system of equations (1) is equivalent to the commutativity condition $[L(p, q), A(p, q)]=0$ for two linear differential operators

$$
L(p, q)=D_{x}-U, \quad A(p, q)=D_{t}-V,
$$

where $D_{x}$ and $D_{t}$ are operators of differentiation in $x$ and $t$ respectively,

$$
U=\lambda J+\left(\begin{array}{rr}
0 & 2 p \\
2 q & 0
\end{array}\right), J=\left(\begin{array}{rr}
1 & 0 \\
0 & -1
\end{array}\right), V=\lambda U-\left(\begin{array}{rr}
2 p q & -p_{x} \\
q_{x} & -2 p q
\end{array}\right),
$$


and $\lambda$ is a spectral parameter. Linear operators $L(p, q), A(p, q)$ form a Lax pair (or a Lax representation) for equation (1).

The NLS system (1) admits an infinite hierarchy of commuting symmetries

$$
p_{t_{k}}=f^{k}, \quad q_{t_{k}}=g^{k}, \quad k=0,1,2, \ldots
$$

where

$$
\begin{array}{rlrl}
f^{0} & =2 p, & g^{0} & =-2 q, \\
f^{1} & =p_{x}, & g^{1} & =q_{x}, \\
f^{2} & =\frac{1}{2} p_{x x}-4 p^{2} q, & g^{2} & =-\frac{1}{2} q_{x x}+4 q^{2} p \quad \text { NLS }, \\
f^{3} & =\frac{1}{4} p_{x x x}-6 p q p_{x}, & g^{3} & =\frac{1}{4} q_{x x}-6 q p q_{x}, \\
f^{4} & =\frac{1}{8} p_{x x x x}-4 p q p_{x x}-3 q p_{x}^{2}-2 p p_{x} q_{x}-p^{2} q_{x x}+12 p^{3} q^{2}, \\
g^{4} & =\frac{1}{8} q_{x x x x}-4 q p q_{x x}-3 p q_{x}^{2}-2 q q_{x} p_{x}-q^{2} p_{x x}+12 q^{3} p^{2}, \ldots
\end{array}
$$

All functions $f^{k}, g^{k} \in\left[\mathbb{C}, p, q ; D_{x}\right]$ are differential polynomials over the field $\mathbb{C}$ of variables $p^{(0)}=p, q^{(0)}=q$ and their $x$-derivatives $p^{(1)}=p_{x}, q^{(1)}=q_{x}, p^{(2)}=p_{x x}, q^{(2)}=q_{x x}, \ldots$ and

$$
D_{x}=\sum_{n=0}^{\infty} p^{(n+1)} \frac{\partial}{\partial p^{(n)}}+q^{(n+1)} \frac{\partial}{\partial q^{(n)}} .
$$

By (generalised) symmetries of the NLS equation (1) we understand derivations

$$
D_{t_{k}}=\sum_{n=0}^{\infty} D_{x}^{n}\left(f^{k}\right) \frac{\partial}{\partial p^{(n)}}+D_{x}^{n}\left(g^{k}\right) \frac{\partial}{\partial q^{(n)}}
$$

commuting with $D_{t}=D_{t_{2}}$ and $D_{x}$

$$
\left[D_{t_{2}}, D_{t_{k}}\right]=0, \quad\left[D_{x}, D_{t_{k}}\right]=0
$$

Symmetries are called commuting if the corresponding derivations commute. It is sufficient to verify that $D_{t_{n}}\left(f_{m}\right)=D_{t_{m}}\left(f_{n}\right)$ and $D_{t_{n}}\left(g_{m}\right)=D_{t_{m}}\left(g_{n}\right)$. Motivations and general definition of symmetries one can find in $[14,15]$.

Each symmetry from this hierarchy has a Lax representation

$$
p_{t_{k}}=f^{k}, \quad q_{t_{k}}=g^{k} \Leftrightarrow\left[L(p, q), A^{k}(p, q)\right]=0
$$

with the same operator $L(p, q)=D_{x}-U$ and $A^{k}(p, q)=D_{t_{k}}-V^{k}$ where matrices $V^{k}$ can be found recursively starting from $V^{0}=J$ and for $k \geq 1$

$$
V^{k+1}=\lambda V^{k}-\frac{1}{2} D_{x}\left(V^{k}\right) J-\frac{1}{2}\left[V^{k}, U\right] J-\frac{1}{2} D_{x}^{-1} \operatorname{Tr}\left(U D_{x}\left(V^{k}\right)\right) J .
$$

Here $D_{x}^{-1}$ stands for integration in $x$. It can be rigorously proven that $\operatorname{Tr}\left(U D_{x}\left(V^{k}\right)\right) \in$ $\operatorname{Im} D_{x}$ for any $k$, thus the integral can be evaluated and the result belongs to the differential ring $\left[\mathbb{C} ; p, q ; D_{x}\right]$. The constants of integration can be chosen arbitrary, or fixed by the condition $\left.V^{k}\right|_{p(x)=q(x)=0}=\lambda^{k} J$. In the latter case

$$
V^{0}=J, V^{1}=U, V^{2}=V, V^{3}=\lambda V^{2}+\frac{1}{2}\left(\begin{array}{cc}
2 p q_{x}-2 q p_{x} & p_{x x}-8 p^{2} q \\
q_{x x}-8 q^{2} p & 2 q p_{x}-2 p q_{x}
\end{array}\right), \ldots
$$


Recursion relation (6) can be simplified and represented in the form

$$
V^{0}=J, \quad V^{n+1}=\lambda V^{n}+B_{n}, \quad n=0,1, \ldots
$$

where the $\lambda$-independent matrices $B_{n}$ can be found recursively

$$
B_{0}=\left(\begin{array}{rr}
0 & 2 p \\
2 q & 0
\end{array}\right), \quad B_{k+1}=\frac{J}{2}\left(D_{x}\left(B_{k}\right)+\left[B_{k}, B_{0}\right]-D_{x}^{-1} \operatorname{Tr}\left(B_{0} D_{x}\left(B_{k}\right)\right)\right) .
$$

The set of Lax operators $\left\{L(p, q), A^{k}(p, q), k \in \mathbb{Z}_{\geq 0}\right\}$ and corresponding compatible partial differential equations (commuting symmetries) $\left\{\left(f^{k}, g^{k}\right), k \in \mathbb{Z}_{\geq 0}\right\}$ form the Lax structure for the Nonlinear Schrödinger equation (1).

\subsection{Darboux and Bäcklund transformations for NLS}

Since all linear differential operators $\left\{L(p, q), A^{k}(p, q), k \in \mathbb{Z}_{\geq 0}\right\}$ commute with each other, there exists a common fundamental solution $\Psi$ of the linear problems

$$
L(p, q) \Psi=0, \quad A^{k}(p, q) \Psi=0 .
$$

We shall study a transformation $\mathcal{S}$ of a fundamental solution

$$
\mathcal{S}: \Psi \mapsto \bar{\Psi}=M \Psi, \quad \operatorname{det} M \not \equiv 0
$$

such that the matrix function $\bar{\Psi}$ is a fundamental solution of the linear problems

$$
L(\bar{p}, \bar{q}) \bar{\Psi}=0, \quad A^{k}(\bar{p}, \bar{q}) \bar{\Psi}=0 .
$$

with new "updated potentials" $\bar{p}, \bar{q}$. In the literature this type of transformation is often referred to as a Darboux transformation and the matrix $M$ is called the Darboux matrix. We shall assume that a Darboux matrix $M$ is a rational function of the spectral parameter $\lambda$, whose entry may depend on $p, q, \bar{p}, \bar{q}$ and may also depend on some auxiliary function(s) $h$ or constant parameter(s) (examples will be given later in this section). The given description of a Darboux transformation can be cast into a rigorous definition using elements of differential-difference ring theory. We wish to avoid the introduction of these concepts at the present time to make the paper accessible to a wider community.

It follows from (7), (8), (9) that transformation $\mathcal{S}$ can be extended to the set of Lax operators:

$$
\begin{aligned}
& \mathcal{S}: L(p, q) \mapsto L(\bar{p}, \bar{q})=M L(p, q) M^{-1}, \\
& \mathcal{S}: A^{k}(p, q) \mapsto A^{k}(\bar{p}, \bar{q})=M A^{k}(p, q) M^{-1}, \quad k=0,1, \ldots .
\end{aligned}
$$

Let us show it for the first equation. Indeed, we have

$$
D_{x} \bar{\Psi}=\mathcal{S}(U) \bar{\Psi} \Rightarrow D_{x}(M \Psi)=\mathcal{S}(U) M \Psi \Rightarrow D_{x}(M)-\mathcal{S}(U) M+M U=0
$$

and thus $L(\bar{p}, \bar{q}) M-M L(p, q)=0$. Here we use notation $\mathcal{S}(U)$ for matrix $U(3)$ in which variables $p, q$ are replaced by $\bar{p}, \bar{q}$. Equations (10) are equivalent to a compatible system of equations for $M$

$$
\begin{aligned}
& D_{x}(M)=\mathcal{S}(U) M-M U, \\
& D_{t_{k}}(M)=\mathcal{S}\left(V^{k}\right) M-M V^{k} .
\end{aligned}
$$


It follows from (10) that a Darboux transformation $\mathcal{S}$ can be regarded as an automorphism of the Lax structure. It maps the set of commuting Lax operators into another commuting set

$$
\mathcal{S}:\left\{L(p, q), A^{k}(p, q) ; k \in \mathbb{Z}_{\geq 0}\right\} \mapsto\left\{L(\bar{p}, \bar{q}), A^{k}(\bar{p}, \bar{q}) ; k \in \mathbb{Z}_{\geq 0}\right\},
$$

and results in a Bäcklund transformation which transforms a solution $p, q$ of the NLS hierarchy into a new solution

$$
\mathcal{S}:(p, q) \mapsto(\bar{p}, \bar{q}) .
$$

Equations (11), (12) follow from the conditions that the map $\mathcal{S}$ and derivations $D_{x}, D_{t_{k}}$ commute

$$
\mathcal{S} L=M L M^{-1}:=\operatorname{Ad}_{M} L, \quad \mathcal{S} A^{k}=M A^{k} M^{-1}:=\operatorname{Ad}_{M} A^{k}, \quad k=0,1, \ldots .
$$

Darboux transformations are obviously invertible and a composition of two Darboux transformations $\mathcal{S}_{1}, \mathcal{S}_{2}$ with Darboux matrices $M_{1}, M_{2}$

$$
\mathcal{S}_{2} \circ \mathcal{S}_{1}: \Psi \mapsto \mathcal{S}_{1}\left(M_{2}\right) M_{1} \Psi
$$

is a Darboux transformation. There is a problem to describe all elementary Darboux transformations for a given Lax structure, such that any rational Darboux transformation can be represented as a composition of the elementary ones. In the cases of the Lax structure corresponding to the Korteweg-de Vries equation and the Nonlinear Schrödinger equation the solution of this problem can be found in [16]. In particular, it has been shown that any Darboux transformation of the Lax operators $L, A(2)$ can be represented as a composition of elementary Darboux transformations $\mathcal{J}_{\beta}, \mathcal{S}_{\alpha}, \mathcal{T}_{h}$ with matrices

$$
\begin{aligned}
& \mathcal{J}_{\beta}: J_{\beta}=\operatorname{diag}\left(\beta, \beta^{-1}\right), \\
& \mathcal{S}_{\alpha}: M_{\alpha}=\left(\begin{array}{cc}
\lambda+p \mathcal{S}_{\alpha}(q)-\alpha & p \\
\mathcal{S}_{\alpha}(q) & 1
\end{array}\right), \\
& \mathcal{T}_{h}: N_{h}=\left(\begin{array}{ll}
\lambda+h & p \\
\mathcal{T}_{h}(q) & 0
\end{array}\right),
\end{aligned}
$$

and their inverse transformations with a certain choice of constant complex parameters $\beta, \alpha$.

It follows from (11) that

$$
\begin{aligned}
& \mathcal{J}_{\beta} L=\operatorname{Ad}_{J_{\beta}} L \Leftrightarrow \mathcal{J}_{\beta}(p)=\beta^{2} p, \mathcal{J}_{\beta}(q)=\beta^{-2} q ; \\
& \mathcal{S}_{\alpha} L=\operatorname{Ad}_{M_{\alpha}} L \Leftrightarrow\left\{\begin{array}{l}
p_{x}=2 \mathcal{S}_{\alpha}(p)-2 p^{2} \mathcal{S}_{\alpha}(q)+2 \alpha p \\
\mathcal{S}_{\alpha}\left(q_{x}\right)=-2 q+2 p \mathcal{S}_{\alpha}\left(q^{2}\right)-2 \alpha \mathcal{S}_{\alpha}(q) ;
\end{array}\right. \\
& \mathcal{T}_{h} L=\operatorname{Ad}_{N_{h}} L \Leftrightarrow\left\{\begin{array}{l}
p_{x}=-2 h p, h_{x}=2\left(\mathcal{T}_{h}-1\right)(p q), \\
p \mathcal{T}_{h}(q)=1 .
\end{array}\right.
\end{aligned}
$$

The first map (16)

$$
\mathcal{T}_{\beta}:(p, q) \mapsto\left(\beta^{2} p, \beta^{-2} q\right)
$$


is nothing but a point symmetry of the NLS equation (1).

Equation (17) is a Bäcklund transformation (the $x$ part of the Bäcklund transformation) for the NLS equation (1). Starting from a solution $p, q$ of the NLS we can find a new solution $\left(p_{1}, q_{1}\right)=\left(\mathcal{S}_{\alpha}(p), \mathcal{S}_{\alpha}(q)\right)$ by solving a Riccati equation for $q_{1}$

$$
q_{1, x}=-2 q+2 p q_{1}^{2}-2 \alpha q_{1}
$$

and then $p_{1}=p^{2} q_{1}-2 \alpha p-p_{x}$. Equations (17) can be regarded as an integrable system of differential-difference equations (D $\Delta \mathrm{Es})$. In variables

$$
p_{n}=\mathcal{S}_{\alpha}^{n}(p), q_{n}=\mathcal{S}_{\alpha}^{n}(q), \alpha_{n}=\mathcal{S}_{\alpha}^{n}(\alpha)
$$

it takes the form [17]

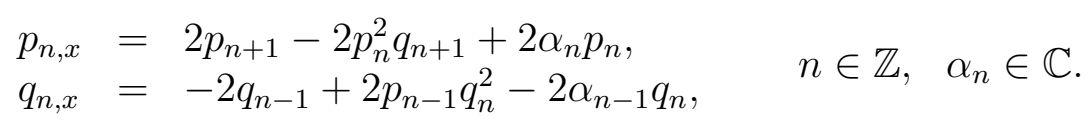

In order to simplify notations we often shall omit $n$ in the lower index for functions depending on the point of the lattice replacing $p_{n \pm 1}$ by $p_{ \pm 1}$, etc. System (20) has a Lax-Darboux representation (often called a semi-discrete Lax representation)

$$
L \Psi=0, \quad \mathcal{S}_{\alpha}(\Psi)=M_{\alpha} \Psi .
$$

Its compatibility condition (11) is equivalent to (20). It has an infinite hierarchy of commuting symmetries following from the conditions $\mathcal{S}_{\alpha} A^{k}=\operatorname{Ad}_{M_{\alpha}} A^{k}$ and local conservation laws. The latter will be shown in the next section.

Automorphism $\mathcal{T}_{h}$ gives the explicit map for solutions of the NLS:

$$
\mathcal{T}_{h}(p)=p^{2} q-\frac{p}{4}\left(\frac{p_{x}}{p}\right)_{x}, \quad \mathcal{T}_{h}(q)=\frac{1}{p} .
$$

In variables $p=\exp \phi, \phi_{k}=\mathcal{T}_{h}^{k}(\phi), h_{k}=\mathcal{T}_{h}^{k}(h)$ it can be written as

$$
\phi_{x}=-2 h, \quad h_{x}=2 \exp \left(\phi_{1}-\phi\right)-2 \exp \left(\phi-\phi_{-1}\right)
$$

and after elimination of $h$ it takes the form of the Toda lattice:

$$
\phi_{x x}=4 \exp \left(\phi-\phi_{-1}\right)-4 \exp \left(\phi_{1}-\phi\right) .
$$

The D $\Delta$ Es which follow from $\mathcal{T}_{h} A^{k}=\operatorname{Ad}_{N_{h}} A^{k}$ are symmetries of (22). For example for $k=0,1,2,3$ we obtain

$$
\begin{aligned}
& \left\{\begin{array} { l } 
{ \phi _ { t _ { 0 } } = 2 , } \\
{ h _ { t _ { 0 } } = 0 ; }
\end{array} \quad \left\{\begin{array}{l}
\phi_{t_{1}}=-2 h \\
h_{t_{1}}=2\left(\mathcal{T}_{h}-1\right) \exp \left(\phi-\phi_{-1}\right) ;
\end{array}\right.\right. \\
& \left\{\begin{array}{l}
\phi_{t_{2}}=2 h^{2}-2\left(\mathcal{T}_{h}+1\right) \exp \left(\phi-\phi_{-1}\right), \\
h_{t_{2}}=-2\left(\mathcal{T}_{h}-1\right)\left(\exp \left(\phi-\phi_{-1}\right)\left(h_{-1}+h\right)\right) ;
\end{array}\right. \\
& \left\{\begin{array}{c}
\phi_{t_{3}}=2 \exp \left(\phi-\phi_{-1}\right)\left(2 h+h_{-1}\right)+2 \exp \left(\phi_{1}-\phi\right)\left(2 h+h_{1}\right)-2 h^{3}, \\
h_{t_{3}}=2\left(\mathcal{T}_{h}-1\right)\left(\left(h_{-1}^{2}+h_{-1} h+h^{2}\right) \exp \left(\phi-\phi_{-1}\right)+\right. \\
\left.\exp \left(2 \phi-2 \phi_{-1}\right)+\left(\mathcal{T}_{h}+1\right) \exp \left(\phi-\phi_{-2}\right)\right) .
\end{array}\right.
\end{aligned}
$$

To define the explicit map (21) we have to consider the localisation of the ring with respect to the element $p^{-1}$. Then we introduced the exponential function in order to transform the system in the standard well known form of the Toda lattice. 


\subsection{Bianchi commutativity of Darboux maps and integrable $\mathrm{P} \Delta \mathrm{Es}$}

Let us impose the condition that automorphisms corresponding to two Darboux maps $\mathcal{S}_{\alpha}$ and $\mathcal{S}_{\beta}$ commute

$$
\left[\mathcal{S}_{\alpha}, \mathcal{S}_{\beta}\right]=0 \quad \Rightarrow \quad \mathcal{S}_{\alpha}\left(M_{\beta}\right) M_{\alpha}-\mathcal{S}_{\beta}\left(M_{\alpha}\right) M_{\beta}=0
$$

It leads to the Bianchi lattice which can be regarded as a system of partial difference equations $(\mathrm{P} \Delta \mathrm{E})$ on $\mathbb{Z}^{2}$.

$$
\left\{\begin{array}{l}
\left(\mathcal{S}_{\beta}(p)-\mathcal{S}_{\alpha}(p)\right)\left(1+p \mathcal{S}_{\beta} \mathcal{S}_{\alpha}(q)\right)-(\alpha-\beta) p=0 \\
\left(\mathcal{S}_{\beta}(q)-\mathcal{S}_{\alpha}(q)\right)\left(1+p \mathcal{S}_{\beta} \mathcal{S}_{\alpha}(q)\right)+(\alpha-\beta) \mathcal{S}_{\beta} \mathcal{S}_{\alpha}(q)=0
\end{array}\right.
$$

Denoting $p_{n m}=\mathcal{S}_{\alpha}^{n} \mathcal{S}_{\beta}^{m}(p), q_{n m}=\mathcal{S}_{\alpha}^{n} \mathcal{S}_{\beta}^{m}(q)$ we get a quadrilateral system of equations:

$$
p_{01}=p_{10}+\frac{\alpha-\beta}{1+p q_{11}} p, q_{01}=q_{10}-\frac{\alpha-\beta}{1+p q_{11}} q_{11} .
$$

$\mathrm{D} \Delta \mathrm{Es}$, which follow from conditions

$$
\mathcal{S}_{\alpha} L=\operatorname{Ad}_{M_{\alpha}} L, \mathcal{S}_{\alpha} A^{k}=\operatorname{Ad}_{M_{\alpha}} A^{k}
$$

are generalised symmetries of (24). Symmetries corresponding to conditions $\mathcal{S}_{\beta} L=$ $\operatorname{Ad}_{M_{\beta}} L, \mathcal{S}_{\beta} A^{k}=\operatorname{Ad}_{M_{\beta}} A^{k}$ are equivalent to (25) modulo system (24).

Similarly, the condition $\left[\mathcal{S}_{\alpha}, \mathcal{T}_{h}\right]=0$ leads to

$$
\mathcal{T}_{h}\left(M_{\alpha}\right) N_{h}=\mathcal{S}_{\alpha}\left(N_{h}\right) M_{\alpha}
$$

which is equivalent to the fully discrete Toda lattice

$$
\mathrm{e}^{\phi_{10-\phi}}-\mathrm{e}^{\phi-\phi_{-10}}+\mathrm{e}^{\phi_{-11}-\phi}-\mathrm{e}^{\phi-\phi_{1,-1}}+\alpha-\alpha_{-1}=0,
$$

and

$$
h=\mathrm{e}^{\phi-\phi_{1,-1}}-\mathrm{e}^{\phi_{10}-\phi}-\alpha,
$$

in the variables $\phi_{p q}=\mathcal{T}_{h}^{p} \mathcal{S}_{\alpha}^{q} \log p$ and $\mathcal{T}_{h}^{k} \alpha=\alpha, \mathcal{S}^{k} \alpha=\alpha_{k}, \alpha_{k} \in \mathbb{C}$. The discrete Toda lattice is a difference equation which is defined on a 5 -points stencil.

Equations which follow from conditions

$$
\mathcal{S}_{\alpha} L=\operatorname{Ad}_{M_{\alpha}} L, \mathcal{S}_{\alpha} A^{k}=\operatorname{Ad}_{M_{\alpha}} A^{k}
$$

are symmetries of the discrete Toda lattice. For example $\mathcal{S}_{\alpha} L=\operatorname{Ad}_{M_{\alpha}} L$ results in

$$
\phi_{x}=-2\left(\mathrm{e}^{\phi-\phi_{1,-1}}-\mathrm{e}^{\phi_{10}-\phi}-\alpha\right) .
$$

Symmetries corresponding to conditions $\mathcal{T}_{h} L=\operatorname{Ad}_{N_{h}} L, \mathcal{T}_{h} A^{k}=\operatorname{Ad}_{N_{h}} A^{k}$ are equivalent to (28) modulo system (27). 


\subsection{Adjacent Lax structure}

In Section 2.2 we discussed the problem to find all elementary Darboux matrices corresponding to a given Lax structure. There is another interesting and important problem to find all possible Lax structures associated with a given Darboux matrix. In this section we show that the Darboux matrix $M_{\alpha}$ (14) corresponding to Lax operators (2) admits an alternative Lax structure with operators $B^{k}=D_{y_{k}}-W^{k}$. We shall assume that $\mathcal{S}_{\alpha}(\alpha)=\alpha$, i.e. the constant $\alpha$ does not depend on the vertex of the lattice.

We notice that the determinant of the Darboux matrix

$$
M_{\alpha}=\left(\begin{array}{cc}
\lambda+p \mathcal{S}_{\alpha}(q)-\alpha & p \\
\mathcal{S}_{\alpha}(q) & 1
\end{array}\right)
$$

is $\lambda-\alpha$. Thus at $\lambda=\alpha M_{\alpha}^{0}=\left.M_{\alpha}\right|_{\lambda=\alpha}$ has rank 1 and can be represented by a bi-vector

$$
M_{\alpha}^{0}=\left(\begin{array}{c}
p \\
1
\end{array}\right) \cdot\left(\mathcal{S}_{\alpha}(q), 1\right)
$$

Let us search for a Lax operator $B_{\alpha}^{1}=D_{y}-W_{\alpha}^{1}$ with a matrix $W_{\alpha}^{1}$ having a simple pole with a residue of rank 1 at $\lambda=\alpha$ and vanishing at $\lambda=\infty$

$$
W_{\alpha}^{1}=\frac{\mathcal{W}_{\alpha}^{1}}{\lambda-\alpha} \text {. }
$$

It follows from $\mathcal{S}_{\alpha} B_{\alpha}^{1}=\operatorname{Ad}_{M_{\alpha}} B_{\alpha}^{1}$ that

$$
D_{y}\left(M_{\alpha}\right)=\mathcal{S}_{\alpha}\left(W_{\alpha}^{1}\right) M_{\alpha}-M_{\alpha} W_{\alpha}^{1} .
$$

Taking the residue at $\lambda=\alpha$ we get equation

$$
S_{\alpha}\left(\mathcal{W}_{\alpha}^{1}\right) M_{\alpha}^{0}=M_{\alpha}^{0} \mathcal{W}_{\alpha}^{1}
$$

which has a unique (up to a scalar constant factor $\gamma$ ) solution

$$
\mathcal{W}_{\alpha}^{1}=\frac{\gamma}{1+\mathcal{S}_{\alpha}^{-1}(p) \mathcal{S}_{\alpha}(q)}\left(\begin{array}{c}
\mathcal{S}_{\alpha}^{-1}(p) \\
1
\end{array}\right) \cdot\left(\mathcal{S}_{\alpha}(q), 1\right) .
$$

In what follows we set $\gamma=1$. Thus

$$
W_{\alpha}^{1}=\frac{1}{(\lambda-\alpha)\left(1+\mathcal{S}_{\alpha}^{-1}(p) \mathcal{S}_{\alpha}(q)\right)}\left(\begin{array}{cc}
\mathcal{S}_{\alpha}^{-1}(p) \mathcal{S}_{\alpha}(q) & \mathcal{S}_{\alpha}^{-1}(p) \\
\mathcal{S}_{\alpha}(q) & 1
\end{array}\right) .
$$

Entries of $W_{\alpha}^{1}$ are not from the differential-difference polynomial ring and localisation of the ring by the element $\left(1+\mathcal{S}_{\alpha}^{-1}(p) \mathcal{S}_{\alpha}(q)\right)^{-1}$ is required.

With this $W_{\alpha}^{1}$ equation (29) is equivalent to the following evolutionary system of integrable differential-difference equations

$$
p_{y}=-\frac{p_{-1}}{1+p_{-1} q_{1}}, q_{y}=\frac{q_{1}}{1+p_{-1}(p) q_{1}} .
$$

Here we use notations introduced in (19). System (30) is a new symmetry of differentialdifference system (20) as well as of partial-difference systems $(24),(27)$. 
It can be shown that there is an infinite hierarchy of commuting operators

$$
B_{\alpha}^{k}=D_{y_{k}}-W_{\alpha}^{k}, \quad W_{\alpha}^{k+1}=\frac{1}{\lambda-\alpha}\left(W_{\alpha}^{k}+C^{k}\right)
$$

and $C^{k}$ is a $\lambda$-independent matrix. Using condition $\left[B_{\alpha}^{1}, B_{\alpha}^{2}\right]=0$ we can find that

$$
C^{1}=\frac{1}{\left(1+p_{-1} q_{1}\right)^{2}}\left(\begin{array}{cc}
p_{-1} q_{1, y}-p_{-1, y} q_{1} & -p_{-1, y}-p_{-1}^{2} q_{1, y} \\
q_{1, y}+q_{-1}^{2} p_{-1, y} & p_{-1, y} q_{1}-p_{-1} q_{1, y}
\end{array}\right) \text {. }
$$

For matrices $W_{\alpha}^{k}$ there exists a recursion (similar to (6)) which enables one to find the infinite hierarchy of operators $B_{\alpha}^{k}$ recursively. Operators $B_{\alpha}^{k}, k=1,2, \ldots$ form the adjacent Lax structure.

The partial differential equation which is equivalent to the condition $\left[B_{\alpha}^{1}, B_{\alpha}^{2}\right]=0$ is of the form

$$
\begin{aligned}
& \left(p_{-1}\right)_{y_{2}}=-\left(p_{-1}\right)_{y y}+\frac{2 q_{1}\left(\left(p_{-1}\right)_{y}\right)^{2}}{1+p_{-1} q_{1}}, \\
& \left(q_{1}\right)_{y_{2}}=\left(q_{1}\right)_{y y}-\frac{2 p_{-1}\left(\left(q_{1}\right)_{y}\right)^{2}}{1+p_{-1} q_{1}} .
\end{aligned}
$$

System (30) is a Bäcklund transformation for (32). Equation (32) is well known, it is a Heisenberg model for ferromagnets [18]

$$
\mathbf{S}_{\tau}=\mathbf{S} \times \mathbf{S}_{y y}, \quad \mathbf{S}^{2}=1
$$

after the change of variable $y_{2}=i \tau$ and stereographic projection

$$
\mathbf{S}=\left(\frac{p_{-1}+q_{1}}{1+p_{-1} q_{1}}, i \frac{q_{1}-p_{-1}}{1+p_{-1} q_{1}}, \frac{p_{-1} q_{1}-1}{1+p_{-1} q_{1}}\right)
$$

We can use equation (30) to eliminate $y$ derivatives from the Lax operator $B_{\alpha}^{2}$ and partial differential equation (32). The latter will take the form of a differential-difference system

$$
\begin{aligned}
& p_{y_{2}}=\frac{p_{-1}^{2} q_{2}\left(1+p_{-2} q\right)-p_{-2}\left(1+p q_{2}\right)}{\left(1+p_{-2} q\right)\left(1+p_{-1} q_{1}\right)^{2}\left(1+p q_{2}\right)}, \\
& q_{y_{2}}=\frac{q_{2}\left(1+p_{-2} q\right)-q_{1}^{2} p_{-2}\left(1+p q_{2}\right)}{\left(1+p_{-2} q\right)\left(1+p_{-1} q_{1}\right)^{2}\left(1+p q_{2}\right)} .
\end{aligned}
$$

Equation (33) is a Bäcklund transformation for (32) and a symmetry of systems (30), (20), (24), (27).

The system of partial difference equations (24) is equivalent to the commutativity of Darboux transformations $\left[\mathcal{S}_{\alpha}, \mathcal{S}_{\beta}\right]=0$, corresponding to Darboux matrices $M_{\alpha}$ and $M_{\beta}$, with distinct values of the parameters $\alpha$ and $\beta$. With these matrices we associate two Lax operators

$$
B_{\alpha}=D_{y}-\frac{W_{\alpha}^{1}}{\lambda-\alpha}, \quad B_{\beta}=D_{z}-\frac{W_{\beta}^{1}}{\lambda-\beta}, \quad \alpha \neq \beta,
$$


which coincide with the Lax pair for the principal chiral field model [19]. The compatibility condition $\left[B_{\alpha}, B_{\beta}\right]=0$ leads to the system

$$
\left(W_{\beta}^{1}\right)_{y}=\frac{\left[W_{\alpha}^{1}, W_{\beta}^{1}\right]}{\beta-\alpha}, \quad\left(W_{\alpha}^{1}\right)_{z}=\frac{\left[W_{\alpha}^{1}, W_{\beta}^{1}\right]}{\beta-\alpha}
$$

which in variables $p_{n m}=\mathcal{S}_{\alpha}^{n} \mathcal{S}_{\beta}^{m}(p), q_{n m}=\mathcal{S}_{\alpha}^{n} \mathcal{S}_{\beta}^{m}(q)$ can be written as

$$
\begin{aligned}
& \left(p_{-1,0}\right)_{z}=\frac{\left(p_{0,-1}-p_{-1,0}\right)\left(1+p_{-1,0} q_{0,1}\right)}{(\alpha-\beta)\left(1+p_{0,-1} q_{0,1}\right)}, \\
& \left(q_{1,0}\right)_{z}=\frac{\left(q_{1,0}-q_{0,1}\right)\left(1+p_{0,-1} q_{1,0}\right)}{(\alpha-\beta)\left(1+p_{0,-1} q_{0,1}\right)} \\
& \left(p_{0,-1}\right)_{y}=\left(p_{-1,0}\right)_{z}, \quad\left(q_{0,1}\right)_{y}=\left(q_{1,0}\right)_{z} .
\end{aligned}
$$

Finally, let us consider the compatibility condition $\left[L, B_{\alpha}\right]=0$ for two linear problems

$$
L \Psi=0, \quad B_{\alpha} \Psi=0
$$

with the original Lax operator $L(2)$ and operator $B_{\alpha}(34)$. Vanishing of the commutator at infinity in $\lambda$ is equivalent to equations (30). Using equations (30) we can express $p_{-1}$ and $q_{1}$ as

$$
p_{-1}=\frac{1+\sqrt{1+4(p)_{y}(q)_{y}}}{2(q)_{y}}, \quad q_{1}=-\frac{1+\sqrt{1+4(p)_{y}(q)_{y}}}{2(p)_{y}} .
$$

Of course there is also the second solution with the negative sign at the square root, it can be treated similarly. Then the compatibility conditions are equivalent to the system of partial differential equations

$$
(p)_{x y}=2 \alpha(p)_{y}+2 p \sqrt{1+4(p)_{y}(q)_{y}}, \quad(q)_{x y}=-2 \alpha(q)_{y}+2 q \sqrt{1+4(p)_{y}(q)_{y}} .
$$

The constant $\alpha$ can be removed by a simple change of variables $P=p e^{-2 \alpha x}, Q=q e^{2 \alpha x}$. Then the system admits an obvious reduction $P=Q$ to a single hyperbolic equation

$$
(P)_{x y}=P \sqrt{1+4\left((P)_{y}\right)^{2}}
$$

The latter equation is well known in the literature. It can be reduced to the sine-Gordon equation by a differential substitution [20]. The above construction provides us with the Lax representation for the system (36) with the Lax operators $L(2)$ and the second operator

$$
B=D_{y}-\frac{1}{2(\lambda-\alpha)}\left(\begin{array}{cc}
\sqrt{1+4(p)_{y}(q)_{y}} & -2(p)_{y} \\
2(q)_{y} & -\sqrt{1+4(p)_{y}(q)_{y}}
\end{array}\right) .
$$

Similarly one can eliminate shifts from operators $B_{\alpha}^{k}$ to build up the Lax structure correponding to (36), (37). 


\section{Formal diagonalisation of the Lax-Darboux Scheme}

In this section we show that the Lax operators and corresponding Darboux matrices can be simultaneously formally diagonalised. The resulting objects will be presented by formal Laurent expansions at poles of the chosen Lax operator. It will enable us to find recursively local conservation laws for corresponding partial differential, differentialdifference and partial difference equations simultaneously. Our aim is to show that for evolutionary equations from the same Lax-Darboux scheme with Lax operators $L, A^{k}, \quad k=0,1, \ldots$ and Darboux maps $\mathcal{S}_{i}, i=1,2, \ldots$ there is an infinite sequence of common local conservation laws with densities $\rho_{n}, r_{n}^{i}$ and fluxes $\sigma_{n}^{k}$. That is,

1. PDE's corresponding to the Lax structure $\left[L, A^{k}\right]=0$ possess conservation lows

$$
D_{t_{k}} \rho_{n}=D_{x} \sigma_{n}^{k}, \quad k=0,1, \ldots
$$

2. For differential-difference equations originating from the conditions $\mathcal{S}_{i} L=\operatorname{Ad}_{M_{i}} L$ and $\mathcal{S}_{i} A^{k}=\operatorname{Ad}_{M_{i}} A^{k}$ there are conservation laws of the form

$$
D_{x} r_{n}^{i}=\left(\mathcal{S}_{i}-1\right) \rho_{n}, \quad D_{t_{k}} r_{n}^{i}=\left(\mathcal{S}_{i}-1\right) \sigma_{n}^{k}
$$

with the same $\rho_{n}$ and $\sigma_{n}^{k}$ as above, modulo equation $\mathcal{S}_{i} L=\operatorname{Ad}_{M_{i}} L$.

3. For partial difference equations, corresponding to the Bianchi lattice $\left[\mathcal{S}_{i}, \mathcal{S}_{j}\right]=0$ the corresponding sequence of the conservation laws are:

$$
\left(\mathcal{S}_{j}-1\right) r_{n}^{i}=\left(\mathcal{S}_{i}-1\right) r_{n}^{j}
$$

We shall demonstrate it on the examples described in the previous section, associated with the Lax-Darboux scheme for the nonlinear Schrödinger equation as well as with the adjacent Lax structures considered in Section 2.4. A generalisation of this approach to other Lax-Darboux schemes (or their parts) often is rather straightforward and it will be discussed at the end of this Section.

\subsection{Formal diagonalisation of the Lax structure for NLS $\left(L, A^{k}\right)$}

In the Lax operator $L(2)$ matrix $U$ has a simple pole in $\lambda$ at infinity with the coefficient $J$ which is diagonal (3). The matrices $V^{k}$ in operators $A^{k}=D_{t_{k}}-V^{k}$ are differential polynomials in variables $p, q$ and their $x$ derivatives with complex coefficients. The leading (in $\lambda$ ) coefficient is also diagonal and is equal to $\lambda^{k} J$. By local functions (in this case) we shall understand elements of the differential polynomial ring $\mathcal{R}_{x}=\left[\mathbb{C} ; p, q ; D_{x}\right]$.

Let us consider endomorphism ad $\operatorname{ad}_{J}$ of the linear space $\mathfrak{M}=\operatorname{Mat}_{2 \times 2}\left(\mathcal{R}_{x}\right)$ of $2 \times 2$ matrices with entries from $\mathcal{R}_{x}$

$$
\operatorname{ad}_{J}: \mathfrak{M} \mapsto \mathfrak{M}, \quad \operatorname{ad}_{J}(a)=J a-a J, \quad a \in \mathfrak{M} .
$$

The kernel of $\operatorname{ad}_{J}$ is the subspace of diagonal matrices, the image space of $\operatorname{ad}_{J}$ is a subspace of off-diagonal matrices. Thus

$$
\mathfrak{M}=\mathfrak{M}_{\|} \oplus \mathfrak{M}_{\perp}, \quad \mathfrak{M}_{\|}=\operatorname{Ker} \operatorname{ad}_{J}, \mathfrak{M}_{\perp}=\operatorname{Imad}_{J}
$$


In $\mathfrak{M}_{\perp}$ the endomorphism ad $\operatorname{ad}_{J}$ is invertible

$$
\operatorname{ad}_{J}^{-1}: \mathfrak{M}_{\perp} \mapsto \mathfrak{M}_{\perp}, \quad \operatorname{ad}_{J}^{-1} a=\frac{1}{4} \operatorname{ad}_{J} a, \quad \forall a \in \mathfrak{M}_{\perp}
$$

In the space $\mathfrak{M}$ it is convenient to introduce two projectors

$$
\pi_{\perp}=\frac{1}{4} \operatorname{ad}_{J}^{2}, \quad \pi_{\|}=i d-\pi_{\perp}
$$

where $i d$ is the identity map. They are projectors on the off-diagonal and diagonal part of a matrix respectively

$$
\pi_{\perp} \mathfrak{M}=\mathfrak{M}_{\perp}, \quad \pi_{\|} \mathfrak{M}=\mathfrak{M}_{\|} .
$$

We shall use a simplified version of the Drinfeld-Sokolov Lemma, which they have formulated and proved in a rather general setting [7].

Lemma 1. For linear operator $L$ (2) there exists a unique formal series

$$
Q=I+\lambda^{-1} Q_{1}+\lambda^{-2} Q_{2}+\lambda^{-3} Q_{3}+\cdots, \quad Q_{k} \in \mathfrak{M}_{\perp}
$$

such that

$$
\mathcal{L}=Q^{-1} L Q=D_{x}-\lambda J-\mathcal{U}_{0}-\lambda^{-1} \mathcal{U}_{1}-\lambda^{-2} \mathcal{U}_{2}-\cdots, \quad \mathcal{U}_{k} \in \mathfrak{M}_{\|}
$$

The coefficients $Q_{k}$ can be found recursively

$$
Q_{1}=-\frac{1}{4} \operatorname{ad}_{J} U, \quad Q_{k+1}=\frac{1}{4} \operatorname{ad}_{J}\left(D_{x}\left(Q_{k}\right)+\sum_{p=1, q=1}^{p+q=k} Q_{p} U Q_{q}\right)
$$

and

$$
\mathcal{U}_{0}=0, \quad \mathcal{U}_{k}=U Q_{k} .
$$

Proof. Let us substitute $L, \mathcal{L}$ and $Q$ in

$$
C=L Q-Q \mathcal{L}=C_{0}+\lambda^{-1} C_{1}+\lambda^{-2} C_{2}+\cdots .
$$

The condition that the formal series $C$ should vanish provides us with a sequence of equations to determine the coefficients $\mathcal{U}_{k}, Q_{k}$. The linear in $\lambda$ term vanishes automatically. The coefficient at $\lambda^{0}$ is

$$
C_{0}=\left[J, Q_{1}\right]+U-\mathcal{U}_{0} .
$$

Applying projectors $\pi_{\|}$and $\pi_{\perp}$ to $C_{0}$ we find that

$$
\pi_{\|}\left(\left[J, Q_{1}\right]+U-\mathcal{U}_{0}\right)=-\mathcal{U}_{0}=0, \quad \pi_{\perp}\left(\left[J, Q_{1}\right]+U-\mathcal{U}_{0}\right)=\left[J, Q_{1}\right]+U=0 .
$$

Thus $\mathcal{U}_{0}=0$ and $Q_{1}=-\frac{1}{4} \operatorname{ad}_{J} U$. The coefficient at $\lambda^{-k}$ is

$$
C_{k}=\operatorname{ad}_{J} Q_{k+1}-D_{x}\left(Q_{k}\right)+U Q_{k}-\mathcal{U}_{k}-\sum_{p=1, q=1}^{p+q=k} Q_{p} \mathcal{U}_{q}
$$


Therefore

$$
\begin{gathered}
\pi_{\|}\left(C_{k}\right)=U Q_{k}-\mathcal{U}_{k}=0 \Rightarrow \mathcal{U}_{k}=U Q_{k}, \\
\pi_{\perp}\left(C_{k}\right)=\operatorname{ad}_{J} Q_{k+1}-D_{x}\left(Q_{k}\right)-\sum_{p=1, q=1}^{p+q=k} Q_{p} \mathcal{U}_{q}=0 .
\end{gathered}
$$

Thus the coefficients $Q_{n}, \mathcal{U}_{n}$ can be found recursively:

$$
Q_{1}=-\frac{1}{4} \operatorname{ad}_{J} U, Q_{k+1}=\frac{1}{4} \operatorname{ad}_{J}\left(D_{x}\left(Q_{k}\right)+\sum_{p=1, q=1}^{p+q=k} Q_{p} U Q_{q}\right), \mathcal{U}_{k}=U Q_{k}
$$

Note that $Q_{k}$ and $\mathcal{U}_{k}$ are all local, i.e. expressed in terms of differential polynomials.

We are going to show that $Q$ diagonalises the whole Lax-Darboux scheme, i.e. diagonalises the operators $A^{k}$ and the Darboux matrices $M_{\alpha}$ (corresponding to $\mathcal{S}_{\alpha}$ ). It is well known that all operators $A^{k}$ in the Lax structure become diagonal and lead to local conservation laws for the corresponding partial differential equations and their symmetries.

Proposition 1. Let $\left[L, A^{k}\right]=0$, where

$$
A^{k}=D_{t_{k}}-\lambda^{k} J-\lambda^{k-1} V_{1-k}^{k}-\lambda^{k-2} V_{2-k}^{k}-\cdots .
$$

Then

$$
\mathcal{A}^{k}=Q^{-1} A^{k} Q=D_{t_{k}}-\lambda^{k} J-\lambda^{k-1} \mathcal{V}_{1-k}^{k}-\lambda^{k-2} \mathcal{V}_{2-k}^{k}+\cdots
$$

has diagonal coefficients $\mathcal{V}_{s}^{k} \in \mathfrak{M}_{\|}, s=k-1, k-2, \ldots$.

Proof. If $\left[L, A^{k}\right]=0$, then $\left[\mathcal{L}, \mathcal{A}^{k}\right]=0$ where $\mathcal{L}=Q^{-1} L Q(39)$ and $\mathcal{A}^{k}=Q^{-1} A^{k} Q$ (41). Using induction we show that all coefficients of the formal series $\mathcal{A}^{k}$ are diagonal. The leading coefficient of the series $\lambda^{k} J$ is diagonal. Let us assume that coefficients $\mathcal{V}_{1-k}^{k}, \mathcal{V}_{2-k}^{k}, \ldots, \mathcal{V}_{n-k}^{k}$ are diagonal. Then the leading term of $\pi_{\perp}\left[\mathcal{L}, \mathcal{A}^{k}\right]$ is equal to $\lambda^{k-n} \operatorname{ad}_{J} \mathcal{V}_{n+1-k}^{k}$. It should vanish and thus $\mathcal{V}_{n+1-k}^{k} \in \mathfrak{M}_{\|}$.

Corollary 1. The following ystems of partial differential equations

$$
\left(p_{t_{k}}=f^{k}, q_{t_{k}}=g^{k}\right) \Leftrightarrow\left[L, A^{k}\right]=0
$$

have an infinite hierarchy of common conservation laws

$$
\left(\mathcal{U}_{n}\right)_{t_{k}}=D_{x}\left(\mathcal{V}_{n}^{k}\right), \quad n=1,2, \ldots
$$

Moreover, $D_{x} \mathcal{V}_{m}^{k}=0$, for $m=1-k, 2-k, \ldots, 1,0$.

Proof. From $\left[L, A^{k}\right]=0$ it follows that $\left[\mathcal{L}, \mathcal{A}^{k}\right]=0$ which leads to

$$
\sum_{n=1}^{\infty} \lambda^{-n}\left(\mathcal{U}_{n}\right)_{t_{k}}-\sum_{n=1-k}^{\infty} \lambda^{-n}\left(\mathcal{V}_{n}^{k}\right)_{x}=0
$$

Vanishing the coefficients at each power $\lambda^{n}$ proves the statement. 
It is easy to show that differential polynomials $\operatorname{Tr} \mathcal{U}_{n} \in \operatorname{Im} D_{x}$ and thus they correspond to trivial densities. Let us take the matrix entry $\left(\mathcal{U}_{n}\right)_{2,2},\left(\mathcal{V}_{n}^{k}\right)_{2,2}$ to define

$$
\rho_{n}=\left(\mathcal{U}_{n}\right)_{2,2}, \quad \sigma_{n}^{k}=\left(\mathcal{V}_{n}^{k}\right)_{2,2} .
$$

It can be shown that the corresponding densities are all non-trivial.

Example. Taking $L$ corresponding to the NLS (2) we find that

$$
\begin{array}{rlrl}
Q_{1} & =\left(\begin{array}{cc}
0 & -p \\
q & 0
\end{array}\right), & \mathcal{U}_{1} & =2 p q\left(\begin{array}{cc}
1 & 0 \\
0 & -1
\end{array}\right), \\
Q_{2}=-\frac{1}{2}\left(\begin{array}{cc}
0 & p_{x} \\
q_{x} & 0
\end{array}\right), & \mathcal{U}_{2}=-\left(\begin{array}{cc}
p q_{x} & 0 \\
0 & q p_{x}
\end{array}\right), \\
Q_{3}=\frac{1}{4}\left(\begin{array}{cc}
0 & -p_{x x}+4 p^{2} q \\
q_{x x}-q^{2} p & 0
\end{array}\right), \mathcal{U}_{3}=\frac{1}{2}\left(\begin{array}{cc}
p q_{x x}-4 p^{2} q^{2} & 0 \\
0 & 4 p^{2} q^{2}-q p_{x x}
\end{array}\right), \ldots
\end{array}
$$

and

$$
\begin{aligned}
& \rho_{1}=-2 p q, \sigma_{1}^{2}=q_{x} p-p_{x} q, \sigma_{1}^{3}=\frac{1}{2}\left(p_{x} q_{x}-p q_{x x}-q p_{x x}\right)+6 p^{2} q^{2} \ldots \\
& \rho_{2}=-q p_{x}, \sigma_{2}^{2}=\frac{1}{2}\left(p_{x} q_{x}-q p_{x x}\right)+2 p^{2} q^{2}, \sigma_{2}^{3}=\frac{1}{4}\left(p_{x x} q_{x}-q p_{x x x}\right)+4 p q^{2} p_{x} \ldots \\
& \left.\rho_{3}=2 p^{2} q^{2}-\frac{1}{2} q p_{x x}, \rho_{4}=p q\left(p q_{x}+4 q p_{x}\right)\right)-\frac{1}{4} q p_{x x x} \ldots
\end{aligned}
$$

\subsection{Formal diagonalisation of the Darboux matrices $M_{\alpha}, N_{h}$}

The diagonalising transformation $Q$ can be extended to the Darboux matrices $M_{\alpha}, M_{\beta}$ and $N_{h}$. We substitute $L=Q \mathcal{L} Q^{-1}$ in $\mathcal{S}_{\alpha}(L)=M_{\alpha} L M_{\alpha}^{-1}$ to obtain

$$
\mathcal{S}_{\alpha}(\mathcal{L})=\mathcal{M}_{\alpha} \mathcal{L} \mathcal{M}_{\alpha}^{-1}, \quad \mathcal{M}_{\alpha}=\mathcal{S}_{\alpha}(Q)^{-1} M_{\alpha} Q
$$

Similarly we obtain

$$
\mathcal{T}_{h}(\mathcal{L})=\mathcal{N}_{h} \mathcal{L N}_{h}^{-1}, \quad \mathcal{N}_{h}=\mathcal{T}_{h}(Q)^{-1} N Q
$$

These equations can be rewritten in the form

$$
\begin{aligned}
& D_{x}\left(\mathcal{M}_{\alpha}\right)=\mathcal{S}_{\alpha}(\mathcal{L}) \mathcal{M}_{\alpha}-\mathcal{M}_{\alpha} \mathcal{L} \\
& D_{x}\left(\mathcal{N}_{h}\right)=\mathcal{T}_{h}(\mathcal{L}) \mathcal{N}_{h}-\mathcal{N}_{h} \mathcal{L}
\end{aligned}
$$

Proposition 2. The coefficients $\mathcal{M}_{\alpha}^{k}, \mathcal{N}_{h}^{k}$ of the formal series

$$
\mathcal{M}_{\alpha}=\mathcal{S}_{\alpha}(Q)^{-1} M_{\alpha} Q=\lambda \mathcal{M}_{\alpha}^{-1}+\mathcal{M}_{\alpha}^{0}+\lambda^{-1} \mathcal{M}_{\alpha}^{1}+\lambda^{-2} \mathcal{M}_{\alpha}^{2}+\cdots
$$

and

$$
\mathcal{N}_{h}=\mathcal{T}_{h}(Q)^{-1} N Q=\lambda \mathcal{N}_{h}^{-1}+\mathcal{N}_{h}^{0}+\lambda^{-1} \mathcal{N}_{h}^{1}+\lambda^{-2} \mathcal{N}_{h}^{2}+\cdots
$$

are diagonal matrices. 
Proof. We prove it by induction. The leading coefficients $\mathcal{M}_{\alpha}^{-1}=\mathcal{N}_{h}-1$ are diagonal matrices with $(1,0)$ on the diagonal. Let us assume that the coefficients $\mathcal{M}_{\alpha}^{0}, \ldots, \mathcal{M}_{\alpha}^{n}$ are diagonal. Taking the coefficient $c_{n}$ at $\lambda^{-n}$ in $D_{x}\left(\mathcal{M}_{\alpha}\right)-\mathcal{S}_{\alpha}(\mathcal{L}) \mathcal{M}_{\alpha}+\mathcal{M}_{\alpha} \mathcal{L}$ we obtain

$$
c_{n}=D_{x}\left(\mathcal{M}_{\alpha}^{n}\right)-\left[J, \mathcal{M}_{\alpha}^{n+1}\right]-\sum_{k=-1}^{n} \mathcal{S}_{\alpha}\left(\mathcal{U}_{n-k}\right) \mathcal{M}_{\alpha}^{k}+\mathcal{M}_{\alpha}^{k} \mathcal{U}_{n-k}=0
$$

Projection $\pi_{\perp}\left(c_{n}\right)=-\left[J, \mathcal{M}_{\alpha}^{n+1}\right]=0$, which implies that $\mathcal{M}_{\alpha}^{n+1}$ is diagonal. The proof is similar for the coefficients of $\mathcal{N}_{h}$.

Equation (43) can be written in the form

$$
D_{x}\left(\log \mathcal{M}_{\alpha}\right)=\left(\mathcal{S}_{\alpha}-I\right) \mathcal{L},
$$

since all matrices in (43) are diagonal. Thus $\log \mathcal{M}_{\alpha}$ is a generating function for local conservation laws for the differential-difference equation (20):

$$
\begin{gathered}
\log \left(\mathcal{M}_{\alpha}\right)_{2,2}=\frac{r_{\alpha}^{1}}{\lambda}+\frac{r_{\alpha}^{2}}{\lambda^{2}}+\frac{r_{\alpha}^{3}}{\lambda^{3}}+\cdots \\
D_{x}\left(r_{\alpha}^{k}\right)=\mathcal{S}_{\alpha}\left(\rho_{k}\right)-\rho_{k} .
\end{gathered}
$$

It follows from (12) and Proposition 1 that

$$
D_{t_{k}}\left(r_{\alpha}^{n}\right)=\mathcal{S}_{\alpha}\left(\sigma_{k}^{n}\right)-\sigma_{k}^{n}
$$

Moreover, it follows from (23) that

$$
\mathcal{S}_{\alpha}\left(\mathcal{M}_{\beta}\right) \mathcal{M}_{\alpha}=\mathcal{S}_{\beta}\left(\mathcal{M}_{\alpha}\right) \mathcal{M}_{\beta}
$$

Therefore

$$
\left(\mathcal{S}_{\alpha}-I\right) \log \mathcal{M}_{\beta}=\left(\mathcal{S}_{\beta}-I\right) \log \mathcal{M}_{\alpha}
$$

and thus

$$
\left(\mathcal{S}_{\alpha}-I\right) r_{\beta}^{k}=\left(\mathcal{S}_{\beta}-I\right) r_{\alpha}^{k}, \quad k=1,2, \ldots
$$

Similarly, from (26) it follows that $\mathcal{T}_{h}\left(\mathcal{M}_{\alpha}\right) \mathcal{N}_{h}=\mathcal{S}_{\alpha}\left(\mathcal{N}_{h}\right) \mathcal{M}_{\alpha}$ and thus

$$
\left(\mathcal{S}_{\alpha}-I\right) r^{k}=\left(\mathcal{T}_{h}-I\right) r_{\alpha}^{k}, \quad \log \left(\mathcal{N}_{h}\right)_{2,2}=\frac{r^{1}}{\lambda}+\frac{r^{2}}{\lambda^{2}}+\frac{r^{3}}{\lambda^{3}}+\cdots
$$

Example. Using equations (17), (18) corresponding to $\mathcal{S}_{\alpha} L=\operatorname{Ad}_{M_{\alpha}} L, \mathcal{T}_{h} L=\operatorname{Ad}_{N} L$ for elimination of all $x$-derivatives we get

$$
\begin{gathered}
Q=I+\lambda^{-1}\left(\begin{array}{cc}
0 & -p \\
q & 0
\end{array}\right)+\lambda^{-2}\left(\begin{array}{cc}
0 & -\alpha p-\mathcal{S}_{\alpha}(p)+p^{2} \mathcal{S}_{\alpha}(q) \\
\mathcal{S}_{\alpha}^{-1}(q)+\alpha q-\mathcal{S}_{\alpha}^{-1}(p) q^{2} & 0
\end{array}\right)+\cdots= \\
I+\lambda^{-1}\left(\begin{array}{cc}
0 & -p \\
q & 0
\end{array}\right)+\lambda^{-2}\left(\begin{array}{cc}
0 & h p \\
-\mathcal{T}_{h}^{-1}\left(h p^{-1}\right) & 0
\end{array}\right)+ \\
+\lambda^{-3}\left(\begin{array}{cc}
0 & -h^{2} p-\mathcal{T}_{h}(p) \\
\mathcal{T}_{h}^{-1}\left(h^{2} p^{-1}\right)-\mathcal{T}_{h}^{-2}\left(p^{-1}\right) & 0
\end{array}\right)+\cdots
\end{gathered}
$$


and

$$
\begin{gathered}
\mathcal{M}_{\alpha}=\lambda\left(\begin{array}{ll}
1 & 0 \\
0 & 0
\end{array}\right)+\left(\begin{array}{cc}
p \mathcal{S}_{\alpha}(q)-\alpha & 0 \\
0 & 1
\end{array}\right)+\lambda^{-1}\left(\begin{array}{cc}
p q & 0 \\
0 & -p \mathcal{S}_{\alpha}(q)
\end{array}\right)+\cdots \\
\mathcal{N}_{h}=\lambda\left(\begin{array}{ll}
1 & 0 \\
0 & 0
\end{array}\right)+\left(\begin{array}{ll}
h & 0 \\
0 & 0
\end{array}\right)+\lambda^{-1}\left(\begin{array}{cc}
p \mathcal{T}_{h}^{-1}\left(p^{-1}\right) & 0 \\
0 & -1
\end{array}\right)+\lambda^{-2}\left(\begin{array}{cc}
-p \mathcal{T}_{h}^{-1}\left(h p^{-1}\right) & 0 \\
0 & h
\end{array}\right)+\cdots
\end{gathered}
$$

Thus

$$
\begin{aligned}
& \rho_{1}=-2 p q=-2 \exp \left(\phi-\mathcal{T}_{h}^{-1} \phi\right), \\
& \rho_{2}=-q p_{x}=-2\left(\alpha p q+\mathcal{S}_{\alpha}(p) q-p^{2} q \mathcal{S}_{\alpha}(q)\right)=2 \exp \left(\phi-\mathcal{T}_{h}^{-1} \phi\right) h \\
& \sigma_{2}^{1}=p q_{x}-q p_{x}, \quad \sigma_{2}^{2}=4 p^{2} q^{2}+\frac{1}{2}\left(p_{x} q_{x}-q p_{x x}\right), \\
& r_{\alpha}^{1}=-p \mathcal{S}_{\alpha}(q), \quad r_{\alpha}^{2}=\frac{1}{2} p^{2} q^{2}-\alpha p \mathcal{S}_{\alpha}(q)-\mathcal{S}_{\alpha}(p q), \\
& r^{1}=-h, \quad r^{2}=\frac{1}{2} h^{2}-\exp \left(\mathcal{T}_{h}(\phi)-\phi\right), \quad r^{3}=-\frac{1}{3} h^{3}+\exp \left(\mathcal{T}_{h}(\phi)-\phi\right)\left(h+\mathcal{T}_{h}(h)\right), \ldots
\end{aligned}
$$

In applications to differential-difference equations one also need to eliminate $x$-derivatives from $\sigma_{2}^{1}, \sigma_{2}^{2}$ using equations (17), (18).

\subsection{Diagonalisation of adjacent Lax structure}

It is easy to justify that the transformation (38), which formally diagonalises the Lax operator $L$ (Lemma 1 ) and operators $A^{k}$, also diagonalises the operators $B_{\alpha}^{k}(31)$ associated with the adjacent Lax structure. For example,

$$
Q^{-1} B_{\alpha}^{1} Q=\frac{\lambda^{-1}}{1+p_{-1} q_{1}}\left(\begin{array}{cc}
p_{-1} q_{1} & 0 \\
0 & 1
\end{array}\right)+\frac{\lambda^{-2}}{1+p_{-1} q_{1}}\left(\begin{array}{cc}
p_{-1}\left(q+\alpha q_{1}\right) & 0 \\
0 & \alpha-p q_{1}
\end{array}\right)+\cdots .
$$

Thus, the coefficients $\hat{\sigma}_{\alpha}^{k}$

$$
\begin{aligned}
& \hat{\sigma}_{\alpha}^{1}=\frac{1}{1+p_{-1} q_{1}}=1-\sqrt{1+4 p_{y} q_{y}}, \\
& \hat{\sigma}_{\alpha}^{2}=\frac{\alpha+p q_{1}}{1+p_{-1} q_{1}}=\alpha\left(1-\sqrt{1+4 p_{y} q_{y}}\right)+p q_{y}, \ldots
\end{aligned}
$$

in the expansion

$$
\left(Q^{-1} B_{\alpha}^{1} Q\right)_{2,2}=\hat{\sigma}_{\alpha}^{1} \lambda^{-1}+\hat{\sigma}_{\alpha}^{2} \lambda^{-2}+\hat{\sigma}_{\alpha}^{3} \lambda^{-3}+\cdots
$$

are fluxes for the local conservation laws of (30)

$$
D_{y}\left(r_{\alpha}^{1}\right)=\left(\mathcal{S}_{\alpha}-1\right) \hat{\sigma}_{\alpha}^{1}, \quad D_{y}\left(r_{\alpha}^{2}\right)=\left(\mathcal{S}_{\alpha}-1\right) \hat{\sigma}_{\alpha}^{2}, \ldots
$$

andfor (36)

$$
D_{y}\left(\rho_{1}\right)=D_{x}\left(\hat{\sigma}_{\alpha}^{1}\right), \quad D_{y}\left(\rho_{2}\right)=D_{x}\left(\hat{\sigma}_{\alpha}^{2}\right), \ldots
$$

where $r_{\alpha}^{1}, r_{\alpha}^{2}$ and $\rho_{1}, \rho_{1}$ are given in (48) and (45),(46) respectively.

Lax operator $B_{\alpha}^{1}$ has a pole at $\lambda=\alpha$ and we can diagonalise it around this pole. It is convenient to introduce a local parameter $\mu=(\lambda-\alpha)^{-1}$ and diagonalise the coefficient at the pole by the gauge transformation

$$
\hat{B}_{\alpha}^{1}=T_{0}^{-1} B_{\alpha}^{1} T_{0}=D_{y}-\mu J_{1}+\hat{W}
$$


where

$$
T_{0}=\left(\begin{array}{cc}
p_{-1} & -1 \\
1 & q_{1}
\end{array}\right), \quad J_{1}=\frac{1}{2}(I+J)=\left(\begin{array}{ll}
1 & 0 \\
0 & 0
\end{array}\right), \hat{W}=\left(\begin{array}{cc}
-q_{1} p_{-1, y} & -q_{1, y} \\
p_{-1, y} & p_{-1} q_{1, y}
\end{array}\right) .
$$

Proposition 3. Transformation $T^{-1} \hat{B} T:=\mathcal{B}$ brings operator $\hat{B}$ (49) to a diagonal form

$$
\mathcal{B}=D_{y}-\mu J_{1}-\mathcal{W}_{0}-\mu^{-1} \mathcal{W}_{1}-\mu^{-2} \mathcal{W}_{2}+\cdots, \quad \mathcal{W}_{k}=\pi_{\|}\left(\mathcal{W}_{k}\right)
$$

Where

$$
\begin{gathered}
\mathcal{W}_{0}=\pi_{\|}(\hat{W}), \quad \mathcal{W}_{k}=\pi_{\perp}(\hat{W}) T_{k} \\
T=I+\mu^{-1} T_{1}+\mu^{-2} T_{2}+\cdots
\end{gathered}
$$

and off-diagonal coefficients $T_{k}$ can be found recursively

$$
\begin{aligned}
& T_{1}=-\frac{1}{2} \operatorname{ad}_{J}(\hat{W}), \\
& T_{k+1}=\frac{1}{2} \operatorname{ad}_{J}\left(T_{k, y}-\pi_{\|}(\hat{W}) T_{k}+T_{k} \pi_{\|}(\hat{W})+\sum_{s=1}^{k-1} T_{k-s} \pi_{\perp}(\hat{W}) T_{s}\right) .
\end{aligned}
$$

We omit the proof since it is very similar to the proof of Lemma 1.

The same transformation $\mathcal{L}=\left(T_{0} T\right)^{-1} L T_{0} T$ brings the Lax operator $L$ to a diagonal form (this diagonalisation is different from the one given in Lemma 1). The coefficients $\varrho_{k}=\left(\mathcal{W}_{k}\right)_{2,2}$ of the expansion

$$
\varrho_{0}=-\frac{p_{-1} q_{1, y}}{1+p_{-1} q_{1}}, \quad \varrho_{1}=-\frac{p_{-1, y} q_{1, y}}{\left(1+p_{-1} q_{1}\right)^{2}}, \ldots
$$

are densities of the conservation laws for the Heisenberg hierarchy (32), principal chiral field model (35) and system (36).

It can be easily shown that transformation $\mathcal{M}_{\alpha}=\mathcal{S}_{\alpha}\left(T_{0} T\right)^{-1} M_{\alpha} T_{0} T$ brings the Darboux matrix $M_{\alpha}$ in a diagonal form $\mathcal{M}_{\alpha}$. To apply the transformation to $M_{\alpha}$ we need to eliminate the $y$-derivatives from the coefficients $T_{k}$ using equation (30).

There is a direct way to diagonalise the Darboux matrix $M_{\alpha}$. Matrix $M_{\alpha}$ has two points on the Riemann sphere, where the leading coefficient (in the local parameter) is singular. Indeed, at $\lambda=\infty$ and $\lambda=\alpha$ the leading coefficients are

$$
\lambda\left(\begin{array}{ll}
1 & 0 \\
0 & 0
\end{array}\right) \text { and }\left(\begin{array}{cc}
p q_{1} & p \\
q_{1} & 1
\end{array}\right)
$$

respectively. Let us diagonalise the Darboux matrix at $\lambda=\alpha$ without using the result Proposition 3. Namely, we can find coefficients of a formal series

$$
T=I+\mu^{-1} T_{1}+\mu^{-2} T_{2}+\mu^{-3} T_{3}+\cdots, \quad T_{k}=\pi_{\perp}\left(T_{k}\right)
$$

such that the coefficients $\tilde{\mathcal{M}}_{k}$ in

$$
\tilde{\mathcal{M}}_{\alpha}=\mathcal{S}_{\alpha}(T)^{-1} \tilde{M}_{\alpha} T=\tilde{\mathcal{M}}_{0}+\mu^{-1} \tilde{\mathcal{M}}_{1}+\mu^{-2} \tilde{\mathcal{M}}_{2}+\cdots, \quad \tilde{\mathcal{M}}_{k}=\pi_{\|}\left(\tilde{\mathcal{M}}_{k}\right)
$$

where

$$
\begin{gathered}
\tilde{M}_{\alpha}=\mathcal{S}_{\alpha}\left(T_{0}\right)^{-1} M_{\alpha} T_{0}=\tilde{M}_{0}+\mu^{-1} \tilde{M}_{1} \\
\tilde{M}_{0}=\left(\begin{array}{cc}
1+p_{-1} q_{1} & 0 \\
0 & 0
\end{array}\right), \tilde{M}_{1}=\frac{1}{1+p q_{2}}\left(\begin{array}{cc}
p_{-1} q_{2} & -q_{2} \\
-p_{-1} & 1
\end{array}\right) .
\end{gathered}
$$


Proposition 4. The coefficients $T_{k}, T_{k}=\pi_{\perp}\left(T_{k}\right)$ such that the coefficients $\tilde{\mathcal{M}}_{0}=$ $\tilde{\mathcal{M}}_{0}, \tilde{\mathcal{M}}_{k+1}=\pi_{\perp}\left(\tilde{M}_{1}\right) T_{k}$ are diagonal can be found recursively

$$
\begin{aligned}
& T_{1}=\frac{1}{\left(1+p_{-1} q_{1}\right)\left(1+p q_{2}\right)}\left(\begin{array}{ll}
0 & q_{2} \\
-p_{-1} & 0
\end{array}\right), \\
& \tilde{\mathcal{M}}_{0} T_{k+1}-\mathcal{S}_{\alpha}\left(T_{k+1}\right) \tilde{\mathcal{M}}_{0}+\pi_{\|}\left(\tilde{M}_{1}\right) T_{k}-\sum_{s=1}^{k} \mathcal{S}_{\alpha}\left(T_{s}\right) \pi_{\perp}\left(\tilde{M}_{1}\right) T_{k-s}=0 .
\end{aligned}
$$

The proof is straightforward. What is important here is that in the recursion we do not need to solve difference equations since $\operatorname{rank} \tilde{\mathcal{M}}_{0}=1$ and $\operatorname{Ker} \tilde{\mathcal{M}}_{0} \bigoplus \operatorname{Im} \tilde{\mathcal{M}}_{0}=$ $\mathbb{C}^{2}$. Therefore all entries of $T_{k}$ and $\tilde{\mathcal{M}}_{k}$ are elements of the difference ring $[\mathbb{C} ; p, q,(1+$ $\left.\left.p_{-1} q_{1}\right)^{1} ; \mathcal{S}_{\alpha}\right]$, i.e difference polynomials of variables $p, q,\left(1+p_{-1} q_{1}\right)^{-1}$ and their $\mathcal{S}_{\alpha}^{k}, k \in \mathbb{Z}$ shifts with complex coefficients.

It follows from Proposition 4 that

$$
\tilde{\mathcal{M}}_{\alpha}=\left(\begin{array}{cc}
1+p_{-1} q_{1} & 0 \\
0 & 0
\end{array}\right)+\frac{\mu^{-1}}{1+p q_{2}}\left(\begin{array}{cc}
p_{-1} q_{2} & 0 \\
0 & 1
\end{array}\right)+\frac{\mu^{-2}}{1+p_{-1} q_{1}}\left(\begin{array}{cc}
\frac{p_{-2} q_{2}}{1+p_{-1} q} & 0 \\
0 & -\frac{p_{-1} q_{2}}{1+p q_{2}}
\end{array}\right)+\cdots
$$

and thus the coefficients $\tilde{r}_{k \alpha}$

$$
\tilde{r}_{\alpha}^{0}=-\log \left(1+p q_{2}\right), \quad \tilde{r}_{\alpha}^{1}=\frac{p_{-1} q_{2}}{\left(1+p q_{2}\right)\left(1+p_{-1} q_{1}\right)}, \ldots
$$

in the expansion of $\left(\tilde{\mathcal{M}}_{\alpha}\right)_{2,2}=-\log (\mu)+\tilde{r}^{0}{ }_{\alpha}+\mu^{-1} \tilde{r}^{1}{ }_{\alpha}+\cdots$ are new densities of local conservation laws for differential difference equations (20), (30) and partial difference equations (24), (27).

It is obvious that the transformation constructed in Proposition 3 and in Proposition 4 coincide modulo equation (30) and these two approaches are equivalent.

\subsection{Summary}

In this paper we have presented the concept of Lax-Darboux scheme and illustrated it on the example of the NLS equation. From the differential-difference algebra point of view the scheme can be described as follows. The base object is a differential-difference ring polynomials $\mathcal{R}=\left[\mathbb{C} ; \mathbf{u} ; D_{x_{1}}, D_{x_{2}}, \ldots ; \mathcal{S}_{1}, \mathcal{S}_{2}, \ldots\right]$ of a (vector) variable $\mathbf{u}=u^{1}, \ldots, u^{M}$, its derivatives and shifts $D_{x_{1}}^{n_{1}} \cdots D_{x_{m}}^{n_{m}} \mathcal{S}_{1}^{s_{1}} \ldots \mathcal{S}_{p}^{s_{p}} \mathbf{u}$, equipped with a set of commuting derivations $D_{x_{k}}, k=1,2, \ldots$ and commuting automorphisms $\mathcal{S}_{i}, i=1,2 \ldots$ To each $D_{x_{k}}$ we associate a Lax operator of the form $L^{k}=D_{x_{k}}-U^{k}$, where $U^{k}$ is $N \times N$ matrix with entries belonging to $\mathcal{R}(\lambda)$, i.e. are rational functions of a spectral parameter $\lambda$ with coefficients from $\mathcal{R}$. With each automorphism $\mathcal{S}_{i}$ we associate a Darboux $N \times N$ matrix $M^{i}$ with entries from $\mathcal{R}(\lambda)$. Then the system of Lax-Darboux equations we identify with the differential-difference ideal

$$
\mathcal{I}=\left\langle\left[L^{i}, L^{j}\right], \mathcal{S}_{i}\left(L^{j}\right)-\operatorname{Ad}_{M^{i}}\left(L^{j}\right), \mathcal{S}_{i}\left(M^{j}\right) M^{i}-\mathcal{S}_{j}\left(M^{j}\right) M^{j}\right\rangle \subset \mathcal{R}
$$

and consider a quotient ring $\mathcal{R}_{\mathcal{I}}=\mathcal{R} / \mathcal{I}$. In this setup a statement that two expressions are equal modulo equations simply means that these two expressions are equal as elements of $\mathcal{R}_{\mathcal{I}}$. 
We can formally diagonalise (or bring to a block-diagonal form) simultaneously all matrices $U^{i}, M^{j}$ near singular points in $\lambda$ and generate infinite sequences $\rho_{k}^{i}, r_{k}^{j}, k=$ $1,2, \ldots$ such that in $\mathcal{R}_{\mathcal{I}}$ they satisfy relations

$$
\begin{aligned}
& D_{x_{n}} \rho_{k}^{j}=D_{x_{j}} \rho_{k}^{n}, \\
& D_{x_{n}} r_{k}^{j}=\left(\mathcal{S}_{j}-1\right) \rho_{k}^{n}, \\
& \left(\mathcal{S}_{i}-1\right) r_{k}^{j}=\left(\mathcal{S}_{j}-1\right) r_{k}^{i} .
\end{aligned}
$$

These relations can be regarded as a sequences of local conservation laws for partial differential, differential difference and partial difference equations. The Lax-Darboux scheme can be generalised to the case when the derivations are not commuting, but such generalisatins are beyond of the scope of this paper.

In the case of the NLS equation the elements of the Lax-Darboux scheme are:

- The Lax structure, i.e. Lax operators $L, A^{k}$ such that the commutativity conditions $\left[L, A^{k}\right]=\left[A^{k}, A^{p}\right]=0$ are equivalent to a system of integrable partial differential equations and its symmetries (5).

- Darboux transformations $\mathcal{S}_{\alpha}, \mathcal{T}_{h}$ with Darboux matrices $M_{\alpha}(14)$ and $N_{h}$ (15) respectively. The compatibility conditions $\mathcal{S}_{\alpha} L=\operatorname{Ad}_{M^{\alpha}} L, \mathcal{T}_{h} L=\operatorname{Ad}_{N_{h}} L$ and $\mathcal{S}_{\alpha} A^{k}=\operatorname{Ad}_{M^{\alpha}} A^{k}, \mathcal{T}_{h} A^{k}=\operatorname{Ad}_{N_{h}} A^{k}$ result in Bäcklund transformations of the above integrable system and its symmetries (20), (21). Bäcklund transformations also can be regarded as integrable differential-difference equations in their own right.

- The Bianchi lattices, which follow from the commutativity conditions for pairs of Darboux transformations result in integrable systems of partial difference equations (24), (27). The mentioned above differential-difference equations (20), (21) are symmetries of these systems.

- There is an adjacent Lax structure (corresponding to operators $B_{\alpha}^{k}(31)$ ) sharing the same Darboux matrix $M_{\alpha}$ and resulting in the differential-difference integrable system (30). The commutativity condition $\left[B_{\alpha}^{1}, B_{\alpha}^{k}\right]=0$ results in the hierarchy of the Heisenberg model (32). The commutativity condition $\left[B_{\alpha}^{1}, B_{\beta}^{1}\right]=0$ is equivalent to the principal chiral field model (35), so that the hierarchy of the Heisenberg equation is a hierarchy of symmetries for (35). Equation $\left[L, B_{\alpha}^{k}\right]=0$ provide us with a hierarchy of symmetries for system (36). Integrable differential-difference systems of equations arising from the conditions $\mathcal{S}_{\alpha}\left(B_{\alpha}^{k}\right)=\operatorname{Ad}_{M_{\alpha}}\left(B_{\alpha}^{k}\right)$ (such as (30) and (33)) are Bäcklund transformations for the above listed hierarchies and symmetries for differential-difference equations (20), (21) and partial difference equations (24), (27).

We have shown that there is a formal diagonalistaion of the Lax-Darboux scheme, i.e. a transformation (in the form of a formal series in the spectral parameter) which diagonalises simultaneously the Lax structure, associated Darboux transformations and adjacent Lax structures. The diagonalised Lax (and adjacent Lax) operators and logarithms of the diagonalised Darboux matrices are generating functions of local conservation laws (both the densities and fluxes) for related partial differential, differential-difference and 
partial difference equations, which are neatly related to each other. Moreover, there may exist several different diagonalisations, which lead to adjacent hierarchies of local conservation laws for equations corresponding to the Lax-Darboux scheme.

\section{Acknowledgements}

I would like to thank participants of my lecture courses, seminars and conferences, where several parts of this study were presented, for their useful comments. In particular I am grateful to G. Berkeley, R.N. Garifullin, S. Konstantinou-Rizos, V.V. Sokolov, R.I. Yamilov and J.P. Wang for discussions and advice. I am gratefully acknowledge support from the Leverhulme Trust.

\section{References}

[1] V.B. Matveev, M.A. Salle, Darboux Transformations and Solitons, Springer Series in Nonlinear Dynamics 4, Springer-Verlag, Berlin, 1991.

[2] C. Rogers, W. K. Schief, "Bäcklund and Darboux transformations", Geometry and modern applications in soliton theory, Cambridge Texts in Applied Mathematics, 2002.

[3] A. I. Bobenko, Yu. B. Suris, "Integrable systems on quad-graphs", Int. Math. Res. Notices, 11, 573-611.

[4] F. Khanizadeh, A. V. Mikhailov, Jing Ping Wang, "Darboux transformations and recursion operators for differential-difference equations", Theoretical and Mathematical Physics, 177(3) (2013), 1606-1654.

[5] A. V. Mikhailov, G. Papamikos, Jing Ping Wang, "Darboux transformation with dihedral reduction group", Journal of Mathematical Physics, 55(11) (2014), 113507, arXiv: 1402.5660 .

[6] W. R. Wasow, Asymptotic expansions of solutions of ordinary differential equations, Pure and applied mathematics, Wiley Interscience Publishes, New York, 1965.

[7] V. G. Drinfel'd, V. V. Sokolov, "Lie algebras and equations of Korteweg- de Vries type", Itogi Nauki i Tekhniki, 24, Akad. Nauk SSSR Vsesoyuz. Inst. Nauchn. i Tekhn. Inform., Moscow, 1984, 81-180.

[8] A. V. Mikhailov, A. B. Shabat, "Conditions for integrability of systems of two equations of the form $u_{t}=A(u) u_{x x}+F\left(u, u_{x}\right)$. I", Teoret. Mat. Fiz., 62(2) (1985), 163-185.

[9] A.V. Mikhailov, "Formal diagonalisation of Darboux transformation and conservation laws of integrable PDEs, PD $\Delta \mathrm{Es}$ and $\mathrm{P} \Delta \mathrm{Es}$ ", International Workshop "Geometric Structures in Integrable Systems" (October 30 November 02, 2012, M.V. Lomonosov Moscow State University, Moscow), http://www.mathnet.ru/php/presentation.phtml?option_lang=eng\&presentid=5934.

[10] A. V. Mikhailov, "Formal diagonalisation of the Lax-Darboux scheme and conservation laws of integrable partial differential, differential-difference and partial difference", DIS A follow-up meeting (8-12 July 2013, Isaac Newton Institute for Mathematical Sciences), http://www.newton.ac.uk/programmes/DIS/seminars/2013071114001.html.

[11] I. T. Habibullin, M. V. Yangubaeva, "Formal diagonalization of a discrete lax operator and conservation laws and symmetries of dynamical systems", Theoretical and Mathematical Physics, 177(3) (2013), 1655-1679.

[12] R. N. Garifullin, A. V. Mikhailov, R. I. Yamilov, "Discrete equation on a square lattice with a nonstandard structure of generalized symmetries", Theoretical and Mathematical Physics, 180(1) (2014), 765-780.

[13] V.E. Zakharov, A. B. Shabat, "Exact theory of two-dimensional self-focusing and onedimensional self-modulation of waves in nonlinear media", Ž. Èksper. Teoret. Fiz., 61(1) (1971), 118-134. 
[14] A. V. Mikhailov, A. B. Shabat, V. V. Sokolov, "The symmetry approach to classification of integrable equations", Springer Ser. Nonlinear Dynamics, Springer, Berlin, 1991, 115-184.

[15] A. V. Mikhailov, editor, "Integrability", Lecture Notes in Physics, 767 (2009).

[16] V.E. Adler, Classification of discrete integrable equations, DSci Thesis, L.D. Landau Institute, 2010.

[17] I. Merola, O. Ragnisco, Gui-Zhang Tu, "A novel hierarchy of integrable lattices", Inverse Problems, 10(6) (1994), 1315-1334.

[18] L. A. Takhtadzhyan, V.E. Zakharov, "Equivalence of the nonlinear Schrödinger equation and the equation of a Heisenberg ferromagnet", Theoretical and Mathematical Physics, 38(1) (1979), 26-35.

[19] V.E. Zakharov, A. V. Mikhailov, "Relativistically invariant two-dimensional models of field theory which are integrable by means of the inverse scattering problem method", $Z h$. Èksper. Teoret. Fiz., 74(6) (1978), 1953-1973.

[20] A. V. Zhiber, V. V. Sokolov, "Exactly integrable hyperbolic equations of Liouville type", Uspekhi Mat. Nauk, 56(1(337)) (2001), 63-106. 
DOI: $10.18255 / 1818-1015-2015-6-795-817$

\title{
Формальная диагонализация схем Лакса-Дарбу
}

\author{
Михайлов А.В. \\ получена 28 ноября 2015
}

\begin{abstract}
В статье мы обсуждаем концепцию схемы Лакса-Дарбу и иллюстрируем ее на хорошо известных примерах, ассоциированных с нелинейным уравнением Шрёдингера. Мы изучаем связи, возникшие благодаря преобразованиям Дарбу, между иерархиями нелинейного уравнения Шрёдингера, модели Гейзенберга, модели главного кирального поля, а также дифференциальноразностными системами (такими как цепочка Тоды и дифференциально-разностная цепочка Гейзенберга) и конечно-разностными интегрируемыми системами. Мы показываем, что существует формальное преобразование, которое одновременно диагонализует все элементы схемы ЛаксаДарбу. Это приводит нас к производящим функциям локальных законов сохранения для всех интегрируемых систем, полученных в рамках данной схемы Лакса-Дарбу. Обсуждаются связи между законами сохранения систем, принадлежащих заданной схеме Лакса-Дарбу.
\end{abstract}

Ключевые слова: формальная диагонализация, схемы Лакса-Дарбу, нелинейное уравнение Шрёдингера

Для цитирования: Михайлов А.В., "Формальная диагонализация схем Лакса-Дарбу", Моделирование и анализ информационных систем, 22:6 (2015), 795-817.

Об авторах:

Михайлов Александр Васильевич - доктор физико-математических наук, профессор,

Школа математики Университета Лидса (Лидс, Великобритания)

University of Leeds, Leeds, LS2 9JT, UK

e-mail: A.V.Mikhailov@ leeds.ac.uk 